$11-1-2005$

\title{
Nonparametric Pooling And Testing Of Preference Ratings For Full-Profile Conjoint Analysis Experiments
}

Rosa Arboretti G.

University of Ferrara

Marco Marozzi

m.marozzi@economia.unife.it

Luigi Salmaso

University of Padua

Follow this and additional works at: http://digitalcommons.wayne.edu/jmasm

Part of the Applied Statistics Commons, Social and Behavioral Sciences Commons, and the Statistical Theory Commons

\section{Recommended Citation}

G., Rosa Arboretti; Marozzi, Marco; and Salmaso, Luigi (2005) "Nonparametric Pooling And Testing Of Preference Ratings For FullProfile Conjoint Analysis Experiments," Journal of Modern Applied Statistical Methods: Vol. 4 : Iss. 2 , Article 20. DOI: $10.22237 /$ jmasm/1130804340

Available at: http://digitalcommons.wayne.edu/jmasm/vol4/iss2/20 


\section{Nonparametric Pooling And Testing Of Preference Ratings For Full-Profile Conjoint Analysis Experiments}

\author{
Rosa Arboretti G. \\ University of Ferrara
}

\author{
Luigi Salmaso \\ University of Padua
}

The problem of pooling customer preference ratings within a conjoint analysis experiment has been addressed. A method based on the nonparametric combination of rankings has been proposed to compete with the usual method based on the arithmetic mean. This method is nonparametric with respect to the underlying dependence structure and so no dependence model must be assumed. The two methods have been compared using Spearman's rank correlation coefficient and related test. Moreover, a further nonparametric testing method has been considered and proposed; this method takes both correlation and distance between ranks into account. By means of a simulation study it has been shown that the NPC Ranking method performs better than the arithmetic mean.

Key words: conjoint analysis, nonparametric inference, nonparametric combination, ranking.

\section{Introduction}

In recent years, there has been a growing level of competitiveness in the offer of products. From a company point of view, one of the conditions of competitive success is a product's high level of correspondence to the varying requirements of the customer (Porter, 1998). Indeed, successful companies invest considerable resources and skills into planning and designing their products in order to incorporate the various requirements of customers into the product itself. The most competitive companies are currently those which use approaches and instruments designed to

Rosa Arboretti Giancristofaro is an Assistant Professor of Statistics. M. Marozzi is a research fellow. L. Salmaso is an Associate Professor of Statistics. This research was conducted at Center for Modeling, Computing and Statistics of the University of Ferrara (CMCS-UNIFE, http://cmcs.unife.it). The main research interests of the authors are nonparametric statistical methods for marketing, business and finance. Send correspondence to M. Marozzi at m.marozzi@economia.unife.it. capture the so-called voice of customer (VOC). In order to do so, companies describe the product idea in terms which the customer can actually perceive. After its definition, the newly developed concept is tested by means of surveys in the field which aim to highlight which characteristics are most important to the customer and what his/her true intentions are in terms of purchasing/fruition. In this way, it is possible to modify the product concept before fully implementing it, in order to maximize adherence to the needs and expectations of potential customers by identifying specific segments of customers. The methods used are generally based on Conjoint Analysis (Dolan, 1993; Gustafsson, Herrmann, \& Huber, 2001).

The term Conjoint Analysis refers to a set of predominantly statistical methodologies which aim to study customer choice models starting with opinions and preferences expressed by customers on various profiles of a product which is going to be developed. Even recent literature on such methodologies is rather fragmented and presents some critical elements, both in terms of the procedure for the definition of the survey design and in terms of the subsequent statistical analysis of gathered data (Gustafsson et al., 2001; Green, Krieger, \& Wind, 2001). In particular, it should be noted that the arithmetic mean (whether weighted or 
not) is mainly used for pooling preference ratings.

One problem that may arise when customer preference ratings are averaged is the so-called majority fallacy (Moore, 1980). This problem occurs when the item chosen by the average customer is not the item chosen most often. For example, if half of the people like large cars and the other half like small ones, the average person would like medium-sized cars, even if no real person wants one. In this article, the problem of pooling preference ratings is addressed. In particular, the Nonparametric Combination of Rankings method (NPC Ranking; Lago \& Pesarin, 2000; Arboretti, 2003) is used and extended. A simulation study is performed to show that the NPC Ranking method performs generally better than the arithmetic mean. To this end, Spearman's rank correlation coefficient is considered and a new nonparametric test $T_{p}$ for ranking comparison is proposed. Furthermore, to study the power of Spearman's $T_{s}$ and $T_{p}$ test in detecting ranking shifts, a further simulation study is performed.

The pooling of preference ratings using the NPC Ranking methodology

In developing a new product/service a company may take $K \geq 2$ attributes (factors) with $P_{1}, P_{2}, \ldots, P_{K}$ values (levels) into consideration. Let $M=\prod_{k=1}^{K} P_{k}$ be the number of possible combinations of levels (treatments). For each treatment (product/service profile) a hypothetical dummy variable is defined as $d_{m k p}=1$, if the level of factor $k$ is $p$ for treatment $m$, otherwise $d_{m k p}=0$. It is assumed that customers assess the overall utility (worth) of a product/service by combining the separate utility value of each attribute. The additive model for total worth of profile $m$ is therefore:

$$
Y_{m}=\sum_{k=1}^{K} \sum_{p=1}^{P_{K}} v_{k p} d_{m k p}+\varepsilon_{m}, m=1, \ldots, M
$$

where the coefficient $v_{k p}$ denotes the part-worth for level $p$ of factor $k$ and $\varepsilon_{1}, \ldots, \varepsilon_{m}$ are iid random residuals with 0 mean and $\sigma^{2}$ variance.

The full-profile method of treatment presentation is considered. Each treatment is described on a profile card. Let us consider $n$ customers who are asked to rate each of $M$ profiles on a scale of 1 to 10. The problem of how to obtain this ranking, i.e. how to pool customer preferences, is addressed in the article. Let $X_{m i}$ be the rate of profile $m$ given by customer $i(i=1, \ldots, n)$. Of course, if $X_{m i}>X_{m^{\prime} i}$, then customer $i$ rates profile $m$ better than profile $m$ '. In the literature this problem is solved by averaging customer ratings $\bar{X}_{m}=\frac{1}{n} \sum_{i=1}^{n} X_{i m}$, $m=1, \quad \ldots, \quad M$, and profile $\tilde{m}$ such that $\bar{X}_{\tilde{m}}=\max \left(\bar{X}_{1}, \ldots, \bar{X}_{M}\right)$ is then the best profile ${ }_{A} R_{\tilde{m}}=M$ (first rank position), profile $\hat{m}$ such that $\bar{X}_{\hat{m}}=\max _{\{i=1, \ldots, M, m \neq \tilde{m}\}}\left(\bar{X}_{1}, \ldots, \bar{X}_{M}\right)$ is the profile with the second rank position ${ }_{A} R_{\hat{m}}=M-1$, and so on. For simplicity's sake, it is assumed that there are no ties in ranking positions.

An alternative way to pool preferences is based on the NPC ranking method (Lago \& Pesarin, 2000). The procedure consists of three steps. In the first step, a score for profile $m$ is computed as follows:

$$
\lambda_{m i}=\frac{\#\left(X_{m i} \geq X_{m^{\prime} i}\right)+0.5}{M+1}
$$

where $\#\left(X_{m i} \geq X_{m^{\prime} i}\right) \quad$ indicates the rank transformation of $X_{m i}$. This step is repeated for each customer $i$ and profile $m$. With respect to relative rank transformation $\#\left(X_{m i} \geq X_{m^{\prime} i}\right) / M$ of $X_{m i}, 0.5$ and 1 have been added respectively to the numerator and the denominator to obtain $\lambda_{m i}$ varying in the open interval $(0,1)$. The reason for such corrections is merely computational, in order to avoid numerical problems with logarithmic transformations later on. Note that the scores $\lambda_{m i}$

are one-to-one increasingly related with the ranks $\#\left(X_{m i} \geq X_{m^{\prime} i}\right)$. By considering $\lambda_{m i}$ s after the first step, it is straightforward to obtain a (partial) ranking of the $M$ profiles for each customer, but it is the global profile rank that is of interest.

In the second step, the scores that customers have assigned to profile $m$ are combined as follows: 


$$
C_{m}=-\sum_{i=1}^{n} \ln \left(1-\lambda_{m i}\right) .
$$

This step is repeated for the remaining $M-1$ profiles and it performs a nonparametric combination of customers' scores. In the last step, the (global) ranking for profile $m$ is computed as ${ }_{B} R_{m}=\#\left(C_{m} \geq C_{m^{\prime}}\right)$. Of course profile $\tilde{m}$ with ${ }_{B} R_{\tilde{m}}=M$ is the first rank position profile, $\hat{m}$ with ${ }_{B} R_{\hat{m}}=M-1$ is the second one, and so on.

It should be noted that Fisher's omnibus combining function is used in the second step. Other possible combining functions are Liptak's $\sum_{i=1}^{n} \Phi^{-1}\left(\lambda_{m i}\right)$, where $\Phi$ is the cumulative distribution function of a standard normal distribution, Tippett's $\max _{i \in\{1, \ldots, n\}}\left(\lambda_{m i}\right)$, the logistic function $\sum_{i=1}^{n} \ln \left(\frac{\lambda_{m i}}{1-\lambda_{m i}}\right)$ and the additive function $\sum_{i=1}^{n} \lambda_{m i}$ (Lago \& Pesarin, 2000). These combining functions (say $\psi$ ) satisfy three properties: argument: $\quad \psi\left(\ldots, \lambda_{m i}, \ldots\right) \geq \psi\left(\ldots, \lambda_{m i}^{\prime}, \ldots\right) \quad$ if $0<\lambda_{m i}^{\prime}<\lambda_{m i}<1$ for whatever $i \in\{1, \ldots, n\}$;

(iii) $\quad \psi$ is symmetric with respect to permutations of the arguments: if $u_{1}, \ldots, u_{n}$ is a permutation of $1, \quad \ldots, \quad n$ then $\psi\left(\lambda_{m 1}, \ldots, \lambda_{m n}\right) \geq \psi\left(\lambda_{m u_{1}}, \ldots, \lambda_{m u_{n}}\right)$

It should also be noted that a central feature of NPC Ranking is the possibility of assigning different degrees of importance to different types of customers. If the company developing the new product/service is more interested in a certain group of customers, it can assign them a weight of $0.5<w<1$ (and weight 1$w$ to the remaining ones). This weighted approach is taken into account in step two of the procedure by computing $-\sum_{i=1}^{n} w_{i} \ln \left(1-\lambda_{m i}\right)$ instead of $-\sum_{i=1}^{n} \ln \left(1-\lambda_{m i}\right)$, where $w_{i}=w$ if customer $i$ belongs to the group of interest and $w_{i}=1-w$ if he does not. It is straightforward to consider more than two weights.

A comparison of preference pooling methods:

Spearman's $I_{s}$ and $I_{p}$ indicators

To show that NPC Ranking generally performs better than the arithmetic mean in pooling preference ratings, a new indicator $I_{p}$ is presented and Spearman's rank correlation coefficient is also considered. Spearman's wellknown correlation coefficient is defined as:

$$
I_{s}=\frac{3 \sum_{m=1}^{M}\left(R_{m}-\pi_{m}\right)^{2}}{M\left(M^{2}-1\right)},
$$

where $R_{m}$ is the observed rank for profile $m$ and $\pi_{m}$ is the reference rank. $I_{s}$ takes values in $[0,1]$ and small values of $I_{s}$ are associated with similar values of $R_{m}$ and $\pi_{m}$. Another indicator is considered:

$$
I_{p}=\sum_{m<m^{\prime}}\left[k_{m m^{\prime}}\left(1+l_{m m^{\prime}}+h_{m m^{\prime}}\right)\right]
$$

where $K_{m m^{\prime}}=1$ when $\left(\pi_{m}-\pi_{m^{\prime}}\right)\left(R_{m}-R_{m^{\prime}}\right)<0$ otherwise $\quad K_{m m^{\prime}}=0, \quad l_{m m^{\prime}}=\left|\pi_{m}-\pi_{m^{\prime}}\right|-1 \quad$ and $h_{m m^{\prime}}=\left|R_{m}-R_{m^{\prime}}\right|-1 . \quad K_{m m^{\prime}}$ takes into account whether or not the observed and reference rankings are coherent (i.e. positive correlated), $l_{m m^{\prime}}\left(h_{m m^{\prime}}\right)$ and it takes into account how far observed (reference) ranks are from each other. Values of $I_{p}$ close to 0 indicate that the observed ranking is very similar to the reference ranking. It is straightforward to show that

$$
0 \leq I_{p} \leq\left[\frac{1}{6} M(M-1)(2 M-1)\right]
$$

and so

$$
\frac{6 \sum_{m<m^{\prime}}\left[k_{m m^{\prime}}\left(1+l_{m m^{\prime}}+h_{m m^{\prime}}\right)\right]}{M(M-1)(2 M-1)} \text { takes values in }[0,1] .
$$


A simulation study has been performed. More precisely, a conjoint analysis experiment with three factors (I, II and III) each with two levels $(+$ and -$)$ is considered. There are $2^{3}=8$ different profiles. It is assumed that the true profile ranking (reference ranking) is known. Consider table 1 , where profile 8 is the best and profile 1 is the worst. Assume the eight profiles are presented to five customers.

Table 1 Reference ranking of profiles

\begin{tabular}{ccccc}
\hline \multirow{2}{*}{ Profile } & \multicolumn{3}{c}{ Factors } & $\begin{array}{c}\text { Preference } \\
\text { Rating }\end{array}$ \\
& I & II & III & \\
\hline 1 & - & - & - & 1 \\
2 & - & - & + & 2 \\
3 & - & + & - & 3 \\
4 & - & + & + & 4 \\
5 & + & - & - & 5 \\
6 & + & - & + & 6 \\
7 & + & + & - & 7 \\
8 & + & + & + & 8 \\
\hline
\end{tabular}

Customer profile ratings are simulated by adding to the reference ranking a random error taken from continuous distributions such as normal $\mathrm{N}(0,1)$, exponential $\exp (1)$, uniform $\mathrm{U}(0,1)$ and Cauchy $\mathrm{Cau}(0,1)$, and from discrete distributions such as binomial $\operatorname{Bi}(8,0.5)$ and Poisson $\mathcal{P}(1): Y_{m i}=\pi_{m}+\varepsilon_{m i}$, where $Y_{m i}$ is the rate of profile $m$ for customer $i, \mu_{m}$ is the reference rank/rate of profile $m\left(\pi_{m}=m\right)$ and $\varepsilon_{m i}$ is the random error denoting the distance between $Y_{m i}$ and the reference value. $\left[Y_{m i}\right], m=1, \ldots, 8$ and $i=1, \ldots, 5$ is a $8 \times 5$ matrix of real numbers. By computing the arithmetic mean or applying the NPC Ranking, two 8x1 vectors of ranks ${ }_{A} \underline{R}$ or ${ }_{B} \underline{R}$ are obtained. 1000 matrixes are randomly generated and 1000 pairs of vectors are then computed. Let ${ }_{A} \underline{R}^{(c)}$ and ${ }_{B} \underline{R}^{(c)}$ indicate the vector of ranks obtained by using the arithmetic mean and the NPC Ranking for simulation $c(c=1, \ldots, 1000)$. Let $\pi^{\prime}=(1,2, \ldots, 8)$. In order to establish which of the two methods is better, Spearman's $I_{s}$ and $I_{p}$ indicators are computed.

More precisely, the two methods are compared using the $I_{p}$ indicator by computing

$$
{ }_{A B} Q_{p}{ }^{\prime}=\#\left(I_{p}\left({ }_{B} \underline{R}^{(c)}, \underline{\pi}\right) \leq I_{p}\left({ }_{A} \underline{R}^{(c)}, \underline{\pi}\right)\right) / 1000,
$$

the proportion of simulations in which $I_{p}\left({ }_{B} \underline{R}^{(c)}, \underline{\pi}\right)$ is less than or equal to $I_{p}\left({ }_{A} \underline{R}^{(c)}, \underline{\pi}\right)$. If this proportion is greater than ${ }_{A B} Q_{p}{ }^{\prime \prime}=\#\left(I_{p}\left({ }_{A} \underline{R}^{(c)}, \underline{\pi}\right) \leq I_{p}\left({ }_{B} \underline{R}^{(c)}, \underline{\pi}\right)\right) / 1000$, then the NPC Ranking method is preferable because rankings obtained using this method are more similar to the reference ranking than those obtained using the arithmetic mean. It is worth noting that ${ }_{A B} Q_{p}{ }^{\prime}{ }_{A B} Q_{p}{ }^{\prime \prime}>1$ because the equalities are counted both in ${ }_{A B} Q_{p}{ }^{\prime}$ and ${ }_{A B} Q_{p}{ }^{\prime \prime}$. A similar comparison is performed by considering the $I_{s}$ indicator and computing ${ }_{A B} Q_{s}{ }^{\prime}=\#\left(I_{s}\left({ }_{B} \underline{R}^{(c)}, \underline{\pi}\right) \leq I_{s}\left({ }_{A} \underline{R}^{(c)}, \underline{\pi}\right)\right) / 1000 \quad$ and ${ }_{A B} Q_{s}{ }^{\prime \prime}=\#\left(I_{s}\left({ }_{A} \underline{R}^{(c)}, \underline{\pi}\right) \leq I_{s}\left({ }_{B} \underline{R}^{(c)}, \underline{\pi}\right)\right) / 1000$. It is also of some interest to compare $I_{p}$ and $I_{s}$ indicators themselves. To this end, ${ }_{p s} Q_{A}{ }^{\prime}$, ${ }_{p s} Q_{A}{ }^{\prime \prime},{ }_{p s} Q_{B}{ }^{\prime}$ and ${ }_{p s} Q_{B}{ }^{\prime \prime}$ are computed as follows:

$$
\begin{gathered}
{ }_{p s} Q_{A}{ }^{\prime}=\#\left(I_{p}\left({ }_{A} \underline{R}^{(c)}, \underline{\pi}\right) \leq I_{s}\left({ }_{A} \underline{R}^{(c)}, \underline{\pi}\right)\right) / 1000, \\
{ }_{p s} Q_{A}{ }^{\prime \prime}=\#\left(I_{s}\left({ }_{A} \underline{R}^{(c)}, \underline{\pi}\right) \leq I_{p}\left({ }_{A} \underline{R}^{(c)}, \underline{\pi}\right)\right) / 1000 \text { and } \\
{ }_{p s} Q_{B}{ }^{\prime}=\#\left(I_{p}\left({ }_{B} \underline{R}^{(c)}, \underline{\pi}\right) \leq I_{s}\left({ }_{B} \underline{R}^{(c)}, \underline{\pi}\right)\right) / 1000, \\
{ }_{p s} Q_{B}{ }^{\prime \prime}=\#\left(I_{s}\left({ }_{B} \underline{R}^{(c)}, \underline{\pi}\right) \leq I_{p}\left({ }_{B} \underline{R}^{(c)}, \underline{\pi}\right)\right) / 1000 .
\end{gathered}
$$

If ${ }_{p s} Q_{A}{ }^{\prime} \geq_{p s} Q_{A}{ }^{\prime \prime}$ then $I_{p}$ is better than $I_{s}$ when the average method is used. If ${ }_{p s} Q_{B}{ }^{\prime} \geq_{p s} Q_{B}{ }^{\prime \prime}$ then $I_{p}$ is better than $I_{s}$ when the NPC Ranking method is used. 
Table 2 Simulation results

\begin{tabular}{ccccccccc}
\hline Distribution & ${ }_{A B} Q_{p}{ }^{\prime}$ & ${ }_{A B} Q_{p}{ }^{\prime \prime}$ & ${ }_{A B} Q_{s}{ }^{\prime}$ & ${ }_{A B} Q_{s}{ }^{\prime \prime}$ & ${ }_{p s} Q_{A}{ }^{\prime}$ & ${ }_{p s} Q_{A}{ }^{\prime \prime}$ & ${ }_{p s} Q_{B}{ }^{\prime}$ & ${ }_{p s} Q_{B}{ }^{\prime \prime}$ \\
\hline Normal & 0.531 & 0.771 & 0.526 & 0.772 & 1.000 & 0.076 & 1.000 & 0.125 \\
Exponential & 0.650 & 0.447 & 0.592 & 0.461 & 0.996 & 0.015 & 0.991 & 0.021 \\
Uniform & 0.441 & 0.757 & 0.439 & 0.758 & 1.000 & 0.109 & 1.000 & 0.017 \\
Cauchy & 0.649 & 0.378 & 0.655 & 0.385 & 0.771 & 0.296 & 0.662 & 0.412 \\
Binomial & 0.559 & 0.487 & 0.600 & 0.436 & 0.844 & 0.196 & 0.906 & 0.112 \\
Poisson & 0.534 & 0.528 & 0.592 & 0.461 & 0.936 & 0.111 & 0.961 & 0.005 \\
\hline
\end{tabular}

As reported in table 2, NPC Ranking is better than the arithmetic mean for Exponential, Cauchy, Binomial and Poisson distributions, using both $I_{p}$ and $I_{s}$ indicators. Only for normal and uniform distributions the arithmetic mean (as can be expected) is better than NPC Ranking. As regards indicator comparisons, $I_{p}$ is clearly better than $I_{s}$ when the arithmetic mean is used as well as when NPC Ranking is used, because ${ }_{p s} Q_{A}{ }^{\prime}$ and ${ }_{p s} Q_{B}{ }^{\prime}$ are greater than ${ }_{p s} Q_{A}{ }^{\prime \prime}$ and ${ }_{p s} Q_{B}{ }^{\prime \prime} \quad$ respectively, for all considered distributions.

In order to obtain further insight into $I_{p}$ and $I_{s}$ indicator comparison, instead of reference ranking $\quad \pi^{\prime}=(1,2,3,4,5,6,7,8), \quad$ ranking $\underline{\gamma^{\prime}}=(1,2,3,6,4,5,7,8)$ has been considered in Monte Carlo simulations. The reference ranking is still $\underline{\pi}$, but now random errors $\varepsilon_{m i}$ are added to $\underline{\gamma}$ and not to $\underline{\pi}$. The power simulation study is set out as follows: indicators $I_{s}$ and $I_{p}$ are considered as test statistics within a permutation framework, i.e.:

$$
T_{p}=\#\left(I_{p}^{*} \geq I_{p}^{o b s}\right) / B
$$

and

$$
T_{s}=\#\left(I_{s}^{*} \geq I_{s}^{o b s}\right) / B
$$

where $I_{p}^{*}$ and $I_{s}^{*}$ are obtained by a random permutation of the observed ranking, $I_{p}^{o b s}$ and $I_{s}^{o b s}$ are the values of indicators $I_{s}$ and $I_{p}$ calculated by comparing the observed ranking with the reference ranking, and $B$ is the number of all possible permutations in a $2^{3}$ factorial design (i.e. $8 !=40320$ permutations).

Tables 3-5 report the results of the simulation study when errors are normal $\mathrm{N}(0,1)$, uniform $\mathrm{U}(0,1)$, exponential $\exp (1)$, Cauchy $\mathrm{Cau}(0,1)$, binomial $\mathrm{Bi}(8,0.5)$ and Poisson $\mathcal{P}(1)$. $T_{s A}$ and $T_{s B}\left(T_{p A}\right.$ and $\left.T_{p B}\right)$ indicate that the test statistic used is in both cases $T_{s}\left(T_{p}\right)$; although the global ranking is obtained either using the arithmetic mean (indicated by the subscript A) or the NPC method (indicated by the subscript B). Simulation results show that a global ranking obtained using the arithmetic mean allows both test statistics $T_{s}$ and $T_{p}$ to gain more power than when the global ranking is obtained using the NPC method, when the underlying error distribution is either normal or uniform. When the error distribution is binomial and Poisson, the power is very similar between the two global ranking procedures.

On the contrary, the power is greater for both $T_{s}$ and $T_{p}$ when the global ranking is obtained using the NPC method when the underlying error distribution is exponential or Cauchy. However, it is important to emphasize that both $T_{s}$ and $T_{p}$ tests are unbiased, because they indicate that the ranking under $H_{1}$ is different with respect to the reference ranking, even when the nominal significance level is very small. Moreover, they are consistent tests (for more details see e.g. Pesarin 2001)

\section{Conclusion}

The problem of pooling customer preference ratings within a conjoint analysis experiment has 
Table 3 Estimated power, normal and uniform error distributions

\begin{tabular}{|c|c|c|c|c|c|c|c|c|}
\hline \multirow[b]{2}{*}{$\alpha$} & \multicolumn{4}{|c|}{ normal } & \multicolumn{4}{|c|}{ uniform } \\
\hline & $T_{s A}$ & $T_{S B}$ & $T_{p A}$ & $T_{p B}$ & $T_{S A}$ & $T_{s B}$ & $T_{p A}$ & $T_{p B}$ \\
\hline 0.010 & 0.030 & 0.032 & 0.032 & 0.038 & 0.163 & 0.135 & 0.163 & 0.135 \\
\hline 0.025 & 0.196 & 0.212 & 0.206 & 0.220 & 0.464 & 0.384 & 0.493 & 0.401 \\
\hline 0.050 & 0.716 & 0.634 & 0.728 & 0.672 & 0.776 & 0.656 & 0.795 & 0.672 \\
\hline 0.075 & 0.896 & 0.838 & 0.896 & 0.836 & 0.907 & 0.811 & 0.907 & 0.813 \\
\hline 0.100 & 0.956 & 0.886 & 0.958 & 0.888 & 0.943 & 0.870 & 0.948 & 0.878 \\
\hline 0.200 & 1.000 & 0.996 & 1.000 & 0.996 & 0.996 & 0.981 & 0.997 & 0.986 \\
\hline 0.300 & 1.000 & 1.000 & 1.000 & 1.000 & 0.999 & 0.994 & 0.999 & 0.995 \\
\hline 0.400 & 1.000 & 1.000 & 1.000 & 1.000 & 1.000 & 0.998 & 1.000 & 0.998 \\
\hline 0.500 & 1.000 & 1.000 & 1.000 & 1.000 & 1.000 & 1.000 & 1.000 & 1.000 \\
\hline 0.600 & 1.000 & 1.000 & 1.000 & 1.000 & 1.000 & 1.000 & 1.000 & 1.000 \\
\hline 0.700 & 1.000 & 1.000 & 1.000 & 1.000 & 1.000 & 1.000 & 1.000 & 1.000 \\
\hline 0.800 & 1.000 & 1.000 & 1.000 & 1.000 & 1.000 & 1.000 & 1.000 & 1.000 \\
\hline 0.900 & 1.000 & 1.000 & 1.000 & 1.000 & 1.000 & 1.000 & 1.000 & 1.000 \\
\hline 1.000 & 1.000 & 1.000 & 1.000 & 1.000 & 1.000 & 1.000 & 1.000 & 1.000 \\
\hline
\end{tabular}

Table 4 Estimated power, exponential and Cauchy error distributions

\begin{tabular}{|c|c|c|c|c|c|c|c|c|}
\hline \multirow[b]{2}{*}{$\alpha$} & \multicolumn{4}{|c|}{ exponential } & \multicolumn{4}{|c|}{ Cauchy } \\
\hline & $T_{S A}$ & $T_{S B}$ & $T_{p A}$ & $T_{p B}$ & $T_{S A}$ & $T_{s B}$ & $T_{p A}$ & $T_{p B}$ \\
\hline 0.010 & 0.017 & 0.020 & 0.017 & 0.020 & 0.054 & 0.139 & 0.054 & 0.139 \\
\hline 0.025 & 0.051 & 0.053 & 0.062 & 0.059 & 0.224 & 0.392 & 0.238 & 0.413 \\
\hline 0.050 & 0.137 & 0.150 & 0.147 & 0.160 & 0.419 & 0.649 & 0.433 & 0.673 \\
\hline 0.075 & 0.218 & 0.220 & 0.220 & 0.221 & 0.537 & 0.805 & 0.538 & 0.806 \\
\hline 0.100 & 0.286 & 0.272 & 0.304 & 0.279 & 0.590 & 0.875 & 0.595 & 0.878 \\
\hline 0.200 & 0.525 & 0.480 & 0.558 & 0.504 & 0.738 & 0.968 & 0.743 & 0.975 \\
\hline 0.300 & 0.652 & 0.625 & 0.675 & 0.647 & 0.819 & 0.990 & 0.823 & 0.991 \\
\hline 0.400 & 0.772 & 0.751 & 0.774 & 0.755 & 0.892 & 0.996 & 0.889 & 0.996 \\
\hline 0.500 & 0.841 & 0.830 & 0.842 & 0.831 & 0.929 & 0.999 & 0.929 & 0.998 \\
\hline 0.600 & 0.898 & 0.883 & 0.902 & 0.895 & 0.955 & 1.000 & 0.959 & 1.000 \\
\hline 0.700 & 0.936 & 0.928 & 0.937 & 0.928 & 0.976 & 1.000 & 0.976 & 1.000 \\
\hline 0.800 & 0.963 & 0.962 & 0.964 & 0.964 & 0.990 & 1.000 & 0.989 & 1.000 \\
\hline 0.900 & 0.986 & 0.984 & 0.987 & 0.984 & 0.996 & 1.000 & 0.996 & 1.000 \\
\hline 1.000 & 1.000 & 1.000 & 1.000 & 1.000 & 1.000 & 1.000 & 1.000 & 1.000 \\
\hline
\end{tabular}


Table 5 Estimated power binomial and Poisson error distributions

\begin{tabular}{|c|c|c|c|c|c|c|c|c|}
\hline \multirow[b]{2}{*}{$\alpha$} & \multicolumn{4}{|c|}{ binomial } & \multicolumn{4}{|c|}{ Poisson } \\
\hline & $T_{S A}$ & $T_{s B}$ & $T_{p A}$ & $T_{p B}$ & $T_{S A}$ & $T_{s B}$ & $T_{p A}$ & $T_{p B}$ \\
\hline 0.010 & 0.492 & 0.408 & 0.506 & 0.409 & 0.012 & 0.020 & 0.018 & 0.020 \\
\hline 0.025 & 0.924 & 0.860 & 0.933 & 0.869 & 0.050 & 0.065 & 0.075 & 0.068 \\
\hline 0.050 & 0.994 & 0.985 & 0.998 & 0.987 & 0.153 & 0.159 & 0.196 & 0.180 \\
\hline 0.075 & 1.000 & 0.998 & 1.000 & 0.999 & 0.244 & 0.264 & 0.273 & 0.266 \\
\hline 0.100 & 1.000 & 0.999 & 1.000 & 0.999 & 0.312 & 0.329 & 0.352 & 0.350 \\
\hline 0.200 & 1.000 & 1.000 & 1.000 & 1.000 & 0.521 & 0.559 & 0.574 & 0.582 \\
\hline 0.300 & 1.000 & 1.000 & 1.000 & 1.000 & 0.656 & 0.681 & 0.699 & 0.696 \\
\hline 0.400 & 1.000 & 1.000 & 1.000 & 1.000 & 0.759 & 0.785 & 0.790 & 0.788 \\
\hline 0.500 & 1.000 & 1.000 & 1.000 & 1.000 & 0.833 & 0.857 & 0.855 & 0.855 \\
\hline 0.600 & 1.000 & 1.000 & 1.000 & 1.000 & 0.902 & 0.897 & 0.923 & 0.908 \\
\hline 0.700 & 1.000 & 1.000 & 1.000 & 1.000 & 0.942 & 0.936 & 0.947 & 0.936 \\
\hline 0.800 & 1.000 & 1.000 & 1.000 & 1.000 & 0.967 & 0.962 & 0.969 & 0.966 \\
\hline 0.900 & 1.000 & 1.000 & 1.000 & 1.000 & 0.985 & 0.993 & 0.987 & 0.994 \\
\hline 1.000 & 1.000 & 1.000 & 1.000 & 1.000 & 1.000 & 1.000 & 1.000 & 1.000 \\
\hline
\end{tabular}

been addressed. A nonparametric method based on the nonparametric combination of rankings has been proposed to compete with the traditional method based on the arithmetic mean. In order to compare these two methods, Spearman's rank correlation coefficient has been considered. Moreover, a further nonparametric method has been considered and proposed. This method takes both correlation and distance between ranks into account. By means of a simulation study, it has been shown that the NPC Ranking method performs better than the arithmetic mean.

The NPC Ranking procedure requires only one assumption in terms of variables, i.e. the inequality $X_{m i} \geq X_{m^{\prime} i}$ means that customer $i$ rates profile $m$ better than profile $m$ '. It should also be noted that a central feature of NPC Ranking is the possibility of assigning different degrees of importance to different types of customers.

Fisher's omnibus combining function has been used. Other combining functions, such as Liptak's, Tippett's, the logistic and additive functions may also be used (for more details see Lago \& Pesarin, 2000).
A power simulation study showed that permutation tests based on $I_{s}$ and $I_{p}$ statistics clearly indicate that the ranking under $H_{1}$ is different with respect to the reference ranking, even when the nominal significance level, chosen for the comparison, is very small.

Within a conjoint analysis experiment, practitioners should take the NPC Ranking method into account for the pooling of customer preference ratings. A computer program to perform the analysis is available at the website http://cmcs.unife.it.

\section{References}

Giancristofaro, R. A. (2003). A new conjoint analysis procedure with application to marketing research. Communications in Statistics - Theory and Methods, 32, 2271-2286.

Dolan, R. J. (1993). Managing the new product development process: Cases and notes. Addison-Wesley: Reading, MA.

Green, P. E., Krieger, A. M., \& Wind, Y. (2001). Thirty years of conjoint analysis: Reflections and prospects. Interfaces, 31(3), S56-S73. 


\section{NONPARAMETRIC POOLING AND TESTING OF PREFERENCE RATINGS}

Gustafsson, A., Herrmann, A., \& Huber, F. (2001). Conjoint measurement: Methods and applications. Springer: Berlin.

Lago, A., \& Pesarin, F. (2000). Nonparametric combination of dependent rankings with application to the quality assessment of industrial products. Metron, LVIII, 39-52.
Moore, W. L. (1980). Levels of aggregation in conjoint analysis: An empirical comparison. Journal of Marketing Research, 11, 516-523.

Porter, M. E. (1998). Competitive strategy: Techniques for analyzing industries and competitors. Free Press: New York. 